\title{
Self-Focused Cognition in Social Anxiety: A Review of the Theoretical and Empirical Literature
}

\author{
Alice R. Norton and Maree J. Abbott \\ Clinical Psychology Unit, School of Psychology, The University of Sydney, New South Wales, Australia
}

\begin{abstract}
Social anxiety disorder (SAD) is characterised by a marked and persistent fear of social or performance situations. Cognitive models suggest that self-focused cognitive processes play a crucial role in generating and maintaining social anxiety, and that self-focused cognition occurs prior to, during, and following social situations (Clark \& Wells, 1995; Rapee \& Heimberg, 1997). There is a substantial body of empirical evidence demonstrating that socially anxious individuals engage in selffocused cognition during and following a social or performance situation. A smaller but growing body literature suggests that a similar process occurs prior to such situations, and that these three processes are interdependent. Furthermore, the vast majority of research to date indicates that self-focused cognitive processes are detrimental, and that they generate and maintain social anxiety in a variety of ways. However, there remains considerable scope for research to further explicate the role of these processes in the maintenance of SAD, and to enhance interventions designed to ameliorate their negative effects.
\end{abstract}

Keywords: social anxiety, self-focused attention, anticipatory rumination, postevent rumination, cognitive model

\section{The Nature of Social Anxiety}

Social anxiety disorder (SAD) involves a marked and persistent irrational fear of being scrutinised or negatively evaluated in interpersonal and/or performance-based social situations (DSM-V; American Psychiatric Association, 2013). In such situations, an individual fears that $\mathrm{s} /$ he will act in a way that will be humiliating or embarrassing and therefore avoids or endures interpersonal and/or performance-based social situations with intense anxiety. Symptoms of social anxiety include cognitive, affective, behavioural, and physiological components (Lehrer \& Woolfolk, 1982), and may be present prior to, during, and following a social or performance situation (Wells \& Clark, 1997).

In Australia, SAD has a 12 -month and lifetime prevalence rate of $4.7 \%$ and $10.6 \%$ respectively, with higher prevalence in females (Australian Bureau of Statistics [ABS], 2007). SAD is the fourth most common psychiatric disorder following harmful alcohol use, post-traumatic stress disorder (PTSD), and depression (ABS, 2007), and is commonly comorbid with other psychiatric disorders (Stein \& Stein, 2008). SAD typically has an early onset (often beginning in childhood or early adolescence) and is considered moderately heritable (Stein \& Stein, 2008). Despite the considerable suffering and functional impairment associated with SAD (including work, social life,

Address for correspondence: Alice Norton, Clinical Psychology Unit (K01), School of Psychology, The University of Sydney NSW 2006, Australia. Email: alice.norton@sydney.edu.au 
family life), fewer than half of individuals with the disorder ever seek treatment, and only do so after 15-20 years of symptoms (Wang et al., 2005).

\section{Self-Focused Cognitive Processes in Models of Social Anxiety}

Cognitive models of social anxiety have identified several mechanisms that account for the persistence of the disorder (Clark \& Wells, 1995; Hofmann, 2007; Rapee $\&$ Heimberg, 1997). Maintaining processes within these models include self-focused attention and the construction of a mental representation of the self as a social object, heightened monitoring of external threat, in-situation safety behaviours and event avoidance, anticipatory and post-event rumination, comparison of one's mental selfrepresentation with appraisal of the audience's expected standard, judgment of the probability and consequence of negative evaluation, and anxiety-induced performance deficits (Clark \& Wells, 1995; Rapee \& Heimberg, 1997). These models suggest that self-focused cognitive processes are key to the generation and maintenance of anxiety experienced in social and performance situations. Specifically, Clark and Wells (1995) suggest that socially anxious individuals engage in self-focused cognition not only during social situations (self-focused attention; SFA), but also prior to (anticipatory rumination; AR) and following social situations (post-event rumination; PER).

\section{Aims and Methodology}

Given the theoretical and empirical significance of self-focused cognitive processes in SAD, this paper aimed to review theoretical models and empirical evidence for these processes, including limitations of current research, suggested future directions, and clinical applications. While SFA has been the subject of two previous reviews (Bögels \& Mansell, 2004; Spurr \& Stopa, 2002), a considerable body of research has been subsequently published, and the current review sought to update this important field. Furthermore, neither review addressed the role of AR or PER. Hence, the current paper aimed to review evidence for self-focused cognitive processes before and after, as well as during social situations, and to explore the relationship between these processes. Importantly, the current paper endeavoured to explore the clinical applications of these findings to support clinicians in providing the most effective treatment to ameliorate the negative impact of self-focused cognitive processes in SAD.

A comprehensive literature search was conducted among articles indexed in PsycInfo, Medline, and PubMed databases. The keywords employed included: social anxiety, SAD, social phobia, self-focus, attention, self-focused attention, anticipatory rumination, anticipatory processing, post-event rumination, post-event processing, theory, intervention, treatment, and attention training. Reference lists of relevant articles were also closely examined for additional papers.

\section{Theoretical Understanding of Self-Focused Cognitive Processes in Social Anxiety}

\section{Self-Focused Attention}

Self-focused attention (SFA) involves awareness of self-referent information, which may present as body state information, thoughts, emotions, beliefs, attitudes, or memories (Ingram, 1990). SFA among socially anxious individuals involves heightened attention toward internal stimuli (e.g., physiological arousal, emotions, cognitions, 


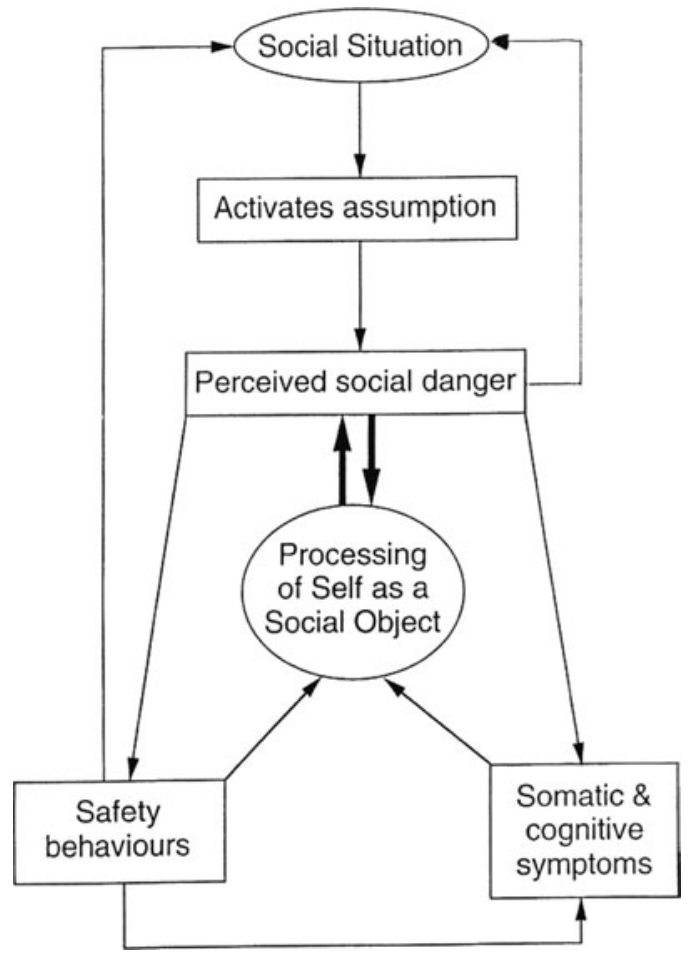

FIGURE 1

Clark's (2001) cognitive model of social anxiety disorder (adapted from Clark \& Wells, 1995). Note: Printed with permission from the publisher, John Wiley \& Sons Ltd.

imagery) during a social or performance situation (Clark \& Wells, 1995; Rapee \& Heimberg, 1997). Thus, Clark and Wells (1995) describe that when socially anxious individuals believe they are in danger of negative evaluation they shift their attention to a process of detailed, self-focused observation, and this increases awareness of self-relevant thoughts, feelings, and internal sensations. This process is intended to facilitate self-preservation by enabling the avoidance of social threat. Once an individual is self-focused, internally generated information is used to construct a mental representation of the individual's appearance and behaviour from an observer perspective (as presumably seen by their audience). This impression of the self is focused on the features of the external self, which may be associated with an increased risk of negative evaluation, and is constructed based on somatic anxiety symptoms (e.g., increased heart rate, sweating, feeling flushed), as well as anxious feelings and cognitions about their social performance (Rapee \& Heimberg, 1997). The mental representation of the self typically incorporates negative images and perceptions of the self in the current social situation, as well as distorted memories of the self in other situations. Rapee and Heimberg (1997) similarly emphasise the role of SFA in maintaining social anxiety, but suggest that socially anxious individuals are simultaneously vigilant to external sources of threat (negative evaluation), such that these attentional processes interact. 


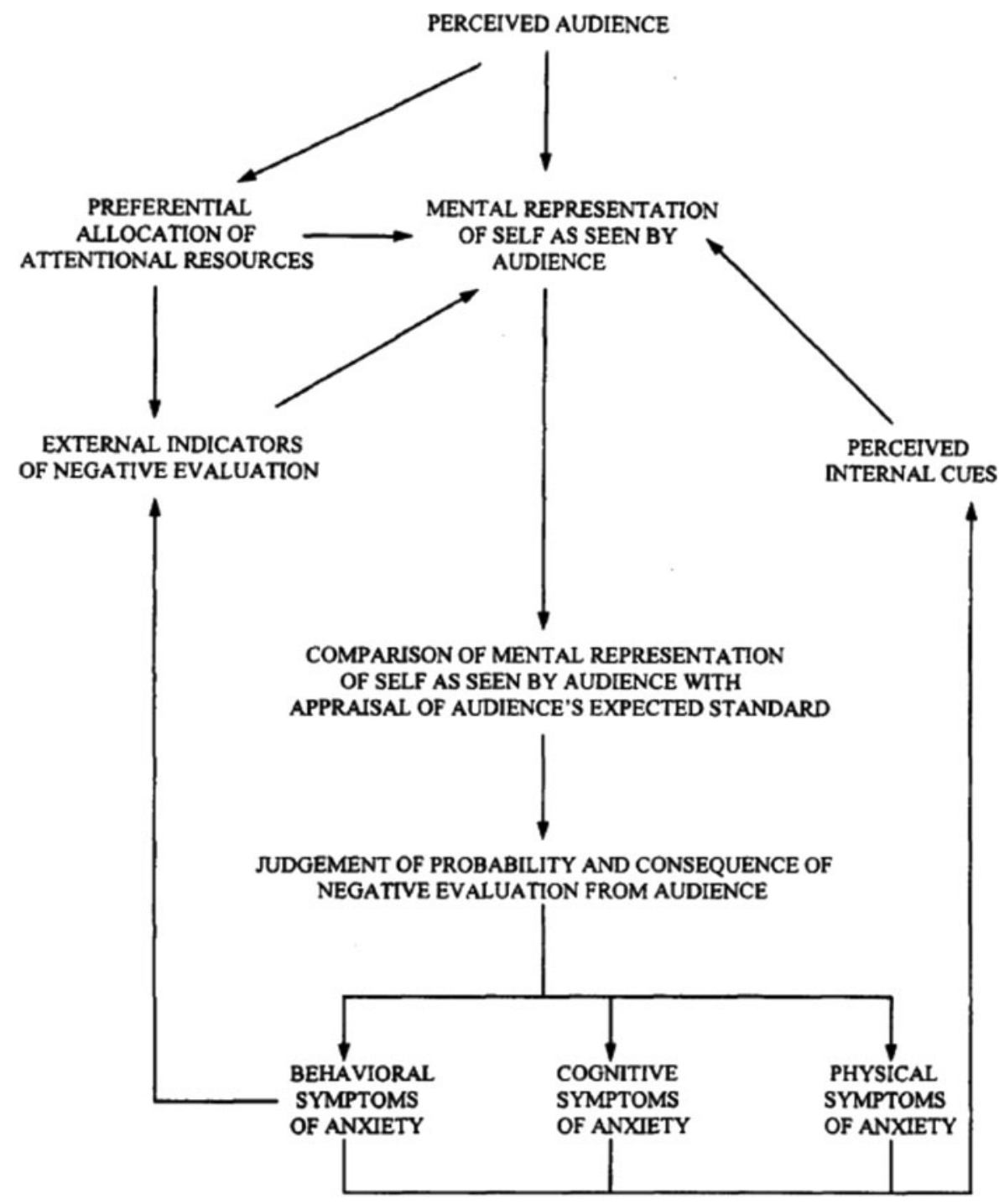

\section{FIGURE 2}

Rapee and Heimberg's (1997) model of social anxiety. Note: Printed with permission from the publisher, Elsevier Science Ltd.

SFA and the use of the observer perspective during social and performance situations is hypothesised to maintain social anxiety in several ways (Clark \& Wells, 1995; Rapee \& Heimberg, 1997). First, SFA exacerbates anxiety by increasing the salience of negative interoceptive information, thoughts, feelings, and self-impressions, leading the individual to sense s/he is being perceived negatively. This occurs at the expense of external information about the social situation, interfering with processing of the audience's behaviour and generating negative biases in the individual's social 
judgments. Thus, the possibility of noticing positive or neutral feedback is reduced, preventing disconfirmation of fears from external information. Moreover, since the elements used to build the impression of the self are symptoms of anxiety, fears of appearing ineffectual or flawed are likely to be confirmed. Taking the observer perspective in constructed self-impressions may further reinforce the individual's belief in the image, as the external viewpoint provides additional credibility. Furthermore, assuming one's own evaluations are also held by others exacerbates the experience of social anxiety. Finally, lack of outward directed attention may further compromise social performance, thereby making feared consequences more likely to occur (Clark $\&$ Wells, 1995; Hofmann, 2007; Rapee \& Heimberg, 1997). Thus, attentional processes are thought to maintain social anxiety by increasing in-situ anxiety and preventing individuals from disconfirming their maladaptive beliefs.

\section{Anticipatory Rumination}

Self-focused cognition is also theorised to be evident in socially anxious individuals prior to a social situation. Anticipatory rumination (AR) is described by Clark and Wells (1995) as dwelling in detail on what might happen in an impending social situation, resulting in biased thinking, as well as influencing anxiety and mood. This 'pre-mortem' leads to heightened anxiety, increased recall of past social failures, and the generation of negative images of one's self in the upcoming situation (based on internal sources of information; e.g., thoughts, feelings, and somatic sensations). This distorted self-image is used to predict how poorly the individual thinks they will perform and how likely they are to be rejected (Clark \& Wells, 1995). Clark and Wells (1995) describe that when socially anxious individuals enter a feared social situation they are 'likely to already be in a self-focused processing mode, to expect outcomes (e.g., physical symptoms) that indicate perceived failure, and to be less likely to notice any signs of being accepted by other people' (p. 74). Consequently, AR is thought to contribute to SAD individuals' in-situ SFA, negative experience of social interaction, poorer social performance, and avoidance behaviours.

\section{Post-Event Rumination}

Clark and Wells (1995) also suggest that processing of the self and performance continues after the conclusion of a social encounter. Post-event rumination (PER) involves a 'post-mortem' in which individuals repetitively dwell on their performance following a social situation (Clark \& Wells, 1995; Rachman, Grüter-Andrew, \& Shafran, 2000; Rapee \& Heimberg, 1997). This review involves reflecting and focusing on anxiety and experience of negative self-perception (actual or perceived inadequacies, mistakes, and imperfections) that occurred during the event, as well as past instances of social failure. Thus, mental representations during PER typically amalgamate negative images/perceptions of the self in the social situation and memories of the self in other social situations (Rapee \& Heimberg, 1997). Moreover, cognitive models suggest that socially anxious individuals selectively attend to negative information about themselves and others during the social situation, subsequently brooding over this negative material, and further distorting or reconstructing the memory over time to fit with their negative self-image (Rapee \& Heimberg, 1997). Consequently, the person comes to perceive the interaction as more negative than it actually was and concludes that the interaction was another social failure, thereby strengthening beliefs about their social inadequacy. Hence, PER is proposed to contribute to the maintenance of 
social anxiety by serving as an intermediate process between an individual's initial interpretations and later recall, thereby fuelling anticipatory anxiety and negative affect, and reinforcing negative beliefs of one's social ability (Brozovich \& Heimberg, 2008; Hofmann, 2007).

\section{The Relationship Between AR, SFA, and PER}

While self-focused cognitive processes are often discussed and investigated independently, it is important to emphasise that they do not operate in isolation. Indeed, cognitive models of social anxiety imply that these processes are interdependent, interacting with one another (and other cognitive processes) in the form of a positive feedback loop. Thus, PER is hypothesised to increase anticipatory anxiety, reactivate negative self-expectations during $\mathrm{AR}$, and contribute to SFA during the situation or avoidance of future social events (Clark \& Wells, 1995). Moreover, SFA during a social situation is believed to distort PER by enhancing memory for negative information. Hence, self-focused cognitive processes that occur prior to, during and following a social event generate a cycle of dysfunctional thinking that contributes to the maintenance of social anxiety (Gaydukevych \& Kocovski, 2012).

\section{Empirical Evidence for Self-Focused Cognitive Processes in Social Anxiety}

A growing body of evidence supports the role of self-focused cognitive processes in generating and maintaining social anxiety.

\section{Self-Focused Attention}

There is considerable support for the role of SFA in the maintenance of SAD, as described by cognitive models (Clark \& Wells, 1995; Rapee \& Heimberg, 1997). First, many studies have found a strong relationship between social anxiety and trait SFA (see Bögels \& Mansell, 2004, for a review). Research has consistently and convincingly demonstrated that clinically and non-clincially socially anxious adults display higher SFA in social situations relative to controls and compared to other phobias (see Bögels \& Mansell, 2004; Hope, Gansler, \& Heimberg, 1989; Spurr \& Stopa, 2002 for reviews), and that these processes also occur among adolescents (Hodson, McManus, Clark, \& Doll, 2008; Ranta, Tuomisto, Kaltiala-Heino, Rantanen, \& Marrunen, 2014) and children (Higa \& Daleiden, 2008; Kley, Tuschen-Caffier, \& Heinrichs, 2011).

Second, studies examining neural substrates, physiological symptomatology and cognitive processes provide robust support for SFA triggered by social threat and associated negative outcomes among socially anxious individuals (Schultz \& Heimberg, 2008). Thus, increased SFA among socially anxious individuals has been correlated with hyperactivation of neural structures related to introspective and self-referential processes, as well as the processing of bodily sensations (Boehme, Miltner, \& Staube, 2015). Moreover, under social/evaluative conditions, socially anxious individuals react faster to internal compared to external stimuli (Mansell, Clark, \& Ehlers, 2003), and report predominantly negative and self-focused cognitions (e.g., Beidel, Turner, \& Dancu, 1985; Dodge, Hope, Heimberg, \& Becker, 1988; Heimberg, Bruch, Hope, \& Dombeck, 1990). In addition, SFA among SAD individuals has been associated with higher state anxiety, more biased and negative self-perceptions, and impaired social performance (e.g., Chen, Rapee, \& Abbott, 2013; Holzman, Valentiner, \& McCraw, 2014; Hope \& Heimberg, 1988; Mahone, Bruch, \& Heimberg, 1993). 
Third, evidence supports the causality of SFA in increasing state anxiety and biased appraisals. This causal relationship has been corroborated by experimentally heightening SFA during social situations and measuring its effect on affective and cognitive processes. A number of studies have demonstrated that inducing SFA via instructions (Woody, 1996; Zou, Hudson, \& Rapee, 2007), the presence of an audience (Woody \& Rodriguez, 2000), the use of mirrors (Bögels, Rijesmus, \& de Jong, 2002), video cameras (Burgio, Merluzzi, \& Prior, 1986; Kashdan \& Roberts, 2004), viewing socially relevant faces (Grisham, King, Makkar, \& Felmingham, 2015), and receiving false physiological feedback indicating an increased heart rate (Makkar \& Grisham, 2013) increases anxiety and avoidance during a social situation, as well as increasing negative performance appraisals and reducing social self-efficacy (Kashdan \& Roberts, 2004).

Fourth, several studies support the contention that negative SFA detracts from external focus on the current social task and prevents socially anxious individuals noticing objective feedback that they could use to update their negative self-impressions and beliefs. After a social situation, socially anxious individuals recall fewer details and therefore have to rely on the biased information they were focused on during the situation (Daly, Vangelisti, \& Lawrence, 1989); thus, they are less accurate in their recall (Hope, Heimberg, \& Klein, 1990) and tend to construe others' reactions to them more negatively (Pozo, Carver, Wellens, \& Scheier, 1991) compared to controls. Conversely, instructing SAD individuals to attend to external information during exposure produces greater reductions in anxiety compared to exposure alone (Wells \& Papageorgiou, 1998).

Fifth, SFA has been associated with negative and biased performance appraisal. Discrepancies between self and observer ratings of performance suggest that socially anxious individuals underestimate their performance and have a distorted representation of how they appear to others (e.g., Abbott \& Rapee, 2004; Mellings \& Alden, 2000; Norton \& Hope, 2001). Indeed, Mellings and Alden (2000) demonstrated that socially anxious participants focused more attention on themselves than their partners during a social interaction and displayed larger negative biases in their self-related judgments compared to controls. These findings are corroborated by demonstrations of impaired attentional control and executive functioning among socially anxious individuals during SFA (Judah, Grant, Mills, \& Lechner, 2013), indicating that self-focus may interfere with ability to accurately interpret social feedback.

Sixth, research supports the role of negative self-imagery and the observer perspective in the maintenance of SAD. SAD individuals are also more likely to report spontaneously occurring negative images during anxiety-provoking social situations and to see these images from an observer perspective (Hackmann, Clark, \& McManus, 2000; Hackmann, Surawy, \& Clark, 1998). Moreover, the tendency of socially anxious individuals to recall social situations from an observer perspective (rather than

50 field perspective - that is, as if looking out through one's own eyes) appears to be specific to situations of high social threat, and becomes more pronounced over time (Coles, Turk, \& Heimberg, 2002; Coles, Turk, Heimberg, \& Fresco, 2001). Indeed, Spurr and Stopa (2003) found that purposefully engaging in an observer perspective (rather than a field perspective) during a social situation resulted in more frequent negative cognitions, more safety behaviours, and worse subjective self-evaluation of performance. Holding negative self-images in mind during a social situation has also been shown to generate greater anxiety, stronger beliefs that one's anxiety symptoms 
are visible, and reduced ratings of subjective performance (Hirsch, Clark, Mathews, \& Willams, 2003).

Finally, in considering empirical support for the role of SFA, it is important to note that Rapee and Heimberg (1997) proposed that SAD individuals focus simultaneously on internal and external cues of threat in social situations, and that these processes are interdependent. They argue that vigilence to external threat informs and increases focus on the internal representation of self, forming a feedback loop that increases state anxiety and maintains SAD. Moreover, the simultaneous allocation of resources to monitoring internal and external stimuli, as well as the task at hand, is considered to impede social performance and may elicit genuine negative evaluation (Heimberg, Bozovich, \& Rapee, 2010). Rapee and Heimberg's (1997) model contrasts with the view of Clark and Wells (1995) that internal self-focus is most signficant in the maintainance of SAD. Thus, Schultz and Heimberg (2008) reviewed evidence for both models, reporting considerable empirical support for the contention that SAD individuals allocate attentional resources to external as well as internal sources of threat. However, the authors note that the findings of most studies (e.g., Mansell, Clark, \& Ehlers, 2003; Pineles \& Mineka, 2005) cannot rule out Clark and Wells' (1995) assertion that SAD individuals may be initially vigilent to external stimuli, but quickly divert their attention away from external sources of social threat (e.g., frowning or bored faces) and almost exclusively focus on internal sources of threat (e.g., somatic symptoms, negative self-imagery). Thus, the impact of external focus of attention on SFA remains unclear, and clarifying the role of focusing on external cues is likely to be important for understanding the maintenance of SAD (Schultz \& Heimberg, 2008).

Nonetheless, the above reviewed studies collectively support cognitive models of SFA in social anxiety, with substantial evidence linking SFA with increased social anxiety, more negative self-judgments, and poorer perception of social performance. The literature indicates that self-focusing in socially anxious individuals increases anxiety by enhancing the processing of self-relevant information, and maintains anxiety via negative interpretive and memory biases that prevent disconfirmation of maladaptive beliefs. Furthermore, reductions in SFA have demonstrated benefits for ameliorating SAD symptomatology. Reductions in SFA during cognitive behaviour therapy (CBT) for SAD have been found to predict reductions in social anxiety and belief in feared outcomes (Furukawa et al., 2009; Schreiber, Heimlich, Schweitzer, \& Stangier, 2015), as well as mediating symptom reduction following both individual and group CBT (Hedman et al., 2013; Mörtberg, Hoffart, Boecking, \& Clark, 2015).

\section{Anticipatory Rumination in Social Anxiety}

Rumination refers to a 'mode of responding to distress that involves repetitively and passively focusing on symptoms of distress and on the possible causes and consequences of these symptoms' (Nolen-Hoeksema, Wisco, \& Lyubomirsky, 2008, p. 400), and is considered a core process in social anxiety (Brozovich \& Heimberg, 2008). Consistent with the extensive evidence regarding the positive relationship between rumination and negative affect (Thomsen, 2006), research indicates that ruminative cognitive processes may be detrimental in social anxiety. Anticipatory rumination (AR) has only recently become a focus of empirical attention; however, a limited body of research supports cognitive models of $A R$ in social anxiety and suggest that it is a 
maintaining factor specific to social anxiety (Mills, Grant, Lechner, \& Judah, 2014). Correlational studies indicate that high socially anxious individuals engage in more AR prior to social or performance situations compared to low socially anxious individuals (Hinrichsen \& Clark, 2003; Penney \& Abbott, 2015; Vassilopoulos, 2004, 2008), and that ruminative thoughts about upcoming social events tend to be recurrent and intrusive, increase anxiety, and interfere with concentration (Vassilopoulos, 2004). Furthermore, consistent with models of AR, highly socially anxious individuals report that they are more likely to dwell on ways of avoiding/escaping social situations, catastrophise about what might happen in the situation, engage in anticipatory safety behaviours, generate negative self-images from an observer perspective, and produce fewer positive autobiographical memories and more negative-evaluative thoughts compared to low socially anxious individuals (Chiupka, Mosovitch, \& Bielak, 2012; Hinrichsen \& Clark, 2003; Vassilopoulos, 2008).

Preliminary experimental studies have compared the effects of induced AR compared to distraction prior to a social task. Some studies have demonstrated that relative to distraction, AR led to increased self-reported and physiological measures (skin conductance) of anxiety, more negative thoughts and unhelpful self-images, predictions of more negative appearance in an impending speech, and stronger conditional and high standard beliefs among individuals high in social anxiety (Brown \& Stopa, 2006; Hinrichsen \& Clark, 2003; Mills, Grant, Lechner et al., 2014; Vassilopoulos, 2005; Wong \& Moulds, 2011). In addition, consistent with Clark \& Wells' (1995) model, AR has been found to increase SFA and focus on somatic information (Mills, Grant, Judah, \& White, 2014), as well as indirectly worsening speech performance via increased self-reported anxiety (Wong \& Moulds, 2011). Correspondingly, the degree to which SAD individuals engage in AR is predicted by anticipatory state anxiety, as well as anticipatory performance and threat appraisal (Penney \& Abbott, 2015).

Furthermore, despite the negative consequences of AR, many socially anxious individuals appear to hold positive metacognitive beliefs regarding the benefits of AR (Vassilopoulos, Brouzos, \& Moberly, 2015), believing that AR is a constructive way of controlling anxiety and handling forthcoming situations more effectively (Vassilopoulos, 2004, 2008). Thus, both low and high socially anxious individuals have reported that memories of past speeches had a helpful influence on speech preparation (Brown \& Stopa, 2006), and some low socially anxious individuals have reported that AR decreased their anxiety (Vassilopoulos, 2004). However, AR has been found to partially mediate the relationship between positive beliefs about AR and social anxiety symptomatology, suggesting that metacognitive beliefs about AR may play a role in the maintenance of social anxiety (Vassilopoulos et al., 2015).

\section{Post-Event Rumination in Social Anxiety}

52 Post-event rumination (PER) has been more extensively studied than AR, with substantive evidence from correlational and experimental research supporting cognitive models of this process in social anxiety (see Brozovich \& Heimberg, 2008). For example, Rachman et al. (2000) observed that socially anxious individuals engaged in more PER about unsatisfactory social events, remembered more negative events with greater frequency, and rated these memories as more intrusive. PER was also correlated with tendency to avoid similar potentially negative social situations (Rachman et al., 2000). Moreover, the positive correlation between social anxiety symptomatology 
and self-reported recall of PER has been replicated by a number of subsequent studies (Fehm, Schneider, \& Hoyer, 2007; Kocovski, Endler, Rector, \& Flett, 2005; Kocovski \& Rector, 2007, 2008; Lundh \& Sperling, 2002; Mellings \& Alden, 2000).

The relationship between social anxiety and PER has also been experimentally examined across social interaction and performance tasks among adults and children. Compared to controls, higher levels of PER have been demonstrated in socially anxious adults the day after a social interaction (Dannahy \& Stopa, 2007; Mellings \& Alden, 2000) and following an impromptu speech task (Edwards, Rapee, \& Franklin, 2003). These findings have been replicated among SAD adults in the week following a speech task (Abbott \& Rapee, 2004; Penny \& Abbott, 2015; Perini, Abbott, \& Rapee, 2006). Furthermore, clinical and subclinical socially anxious children demonstrate more PER than controls following exposure to a social-evaluative situation, and self-appraisals worsened over time (Schmitz, Kramer, Blechert, \& Tuschen-Caffier, 2010; Schmitz, Krämer, \& Tuschen-Caffier, 2011). These preliminary findings indicate that PER may have an early onset, and suggest that it is relevant to the maintenance of SAD across the lifespan.

Other aspects of cognitive models of PER are also supported by the literature, with studies suggesting that PER is predicted by, and maintains, negative self-perceptions. Dysfunctional cognitions, biased threat appraisals, and negative self-evaluations have been demonstrated to be key determinants of PER (Gramer, Schild, \& Lurz, 2012; Kiko et al., 2012; Makkar \& Grisham, 2011a; Penney \& Abbott, 2015; Perini et al., 2006; Zou \& Abbott, 2012). Moreover, socially anxious individuals report more frequent, intense, and longer PER following negative-evaluative social events compared to guilt- or anger-inducing events (Lundh \& Sperling, 2002), as well as situations involving social compared to non-social threat (Fehm et al., 2007). Frequency of PER has also been shown to predict recall of negative self-related information on an open-ended memory task (Mellings \& Alden, 2000). During PER, socially anxious individuals report thought content that serves to reinforce negative perceptions of performance, including more negative and upward counterfactual thoughts ('if only' type thoughts about how the situation could have been different; Kocovski et al., 2005), and more thoughts concerned with poor presentation (e.g., poor posture; Kocovski, MacKenzie, \& Rector, 2011). Furthermore, high socially anxious individuals report more negative self-images associated with higher negative affect and more negative self-related beliefs relative to low socially anxious individuals (Chiupka et al., 2012). Such use of imagery during PER may be particularly detrimental for socially anxious individuals, with a recent study finding that compared to semantic-PER, PER involving imagery induced more anxiety in anticipation of a speech task, as well as more negatively biased interpretations of ambiguous social situations (Brozovich \& Heimberg, 2013).

Hence, the literature supports theories of PER as a cognitive process that perpetuates social anxiety by maintaining negative impressions of one's self, negative memories of social situations, and negative assumptions of future social events (Brozovich \& Heimberg, 2008). Furthermore, PER appears to have lasting sequelae. SAD individuals who engage in PER maintain negative views of their performance for up to 3 weeks, whereas non-anxious controls demonstrated increased positivity in regard to their performance (Abbott \& Rapee, 2004). In addition, compared to non-anxious controls, socially anxious individuals with a higher trait tendency to engage in PER evaluate their performance on a social interaction more negatively a week after its occurrence (Brozovich \& Heimberg, 2011). 
However, positive metacognitive beliefs about the utility of PER have been associated with PER and social anxiety symptomatology, suggesting that SAD individuals may not consider this process to be detrimental, thereby perpetuating their tendency to engage in PER (Fisak \& Hammond, 2013). Indeed, in contrast with cognitive models of SAD, Wong and colleagues (2015) report findings indicating that PER may not be maladaptive, allowing socially anxious individuals to rationalise perceived social failure. Unexpectedly, in a group with high social anxiety and a high number of recent social stressors, those reporting high levels of PER demonstrated no increase in dysfunctional beliefs over the subsequent week, whereas those reporting low levels of PER demonstrated an increase in dysfunctional beliefs (Wong, McEvoy, \& Rapee, 2015). The authors alternatively suggest that this pattern may only occur in non-clinical samples, or that those reporting a low number of thoughts about the past may have been engaging in avoidance and thought suppression, which has been found to increase symptomatology (e.g., Salters-Pedneult, Tull, \& Roemer, 2004).

Nonetheless, consistent with studies of depressive rumination, some evidence suggests that distraction may attenuate the effects of PER. Blagden and Craske (1996) demonstrated that rumination prolonged negative affect following an anxiety induction, whereas distraction significantly reduced anxiety. Moreover, socially anxious individuals report less anxiety, more positive thoughts, and weaker unconditional beliefs (e.g., 'People will think I'm inferior') when instructed to distract compared to ruminate following a speech task (Kocovski et al., 2011; Wong \& Moulds, 2009). However, support for the utility of distraction in reducing social anxiety is inconsistent. Cassin and Rector (2011) reported that distraction did not reduce distress following PER compared to a control condition. Furthermore, Field and Morgan (2004) found that socially anxious participants recalled more negative and shameful memories regardless of whether they were instructed to ruminate or distract. These findings are consistent with Watkins and Teasdale's (2004) proposition that deliberately redirecting attention away from negative thoughts and feelings by focusing on non-self-related information may not be an optimal strategy for dealing with negative affective states (anxiety or depression). Watkins and Teasdale (2004) contend that distraction is a resource-intensive process, and may also reinforce unhelpful cognitive patterns that paradoxically increase negative affect (e.g., thought suppression, Fehm \& Margraf, 2002; and experiential avoidance, Hayes, Wilson, Strosahl, Gifford, \& Follette, 1996). Moreover, focusing attention away from negative thoughts and feelings impedes the development of self-insight or accurate cognitive representations of one's experiences (Watkins \& Teasdale, 2004).

\section{The Relationship Between AR, SFA, and PER}

While cognitive models of social anxiety imply that these self-focused cognitive processes are interdependent, very few studies to date have examined the relationship between these processes, and all have focused on the impact of SFA on PER. Preliminary findings suggest that SFA on a negative self-image (compared to a neutral self-image) during a social encounter predicts more negative and less positive PER (Makkar \& Grisham, 2011b) and that inducing high SFA leads to more frequent PER in the following 24 hours (Gaydukevych \& Kocovski, 2012). Likewise, structural equation modelling suggests that PER is partially predicted by the degree to which in-vivo SFA is focused on a negative self-image (Chen et al., 2013). 


\section{Limitations in Prior Research}

As demonstrated, empirical studies predominantly support self-focused cognitive processes of SAD and suggest that SFA, AR, and PER play a significant role in the maintenance of the disorder. However, there are a number of methodological limitations in the literature to date. Importantly, many experimental studies fail to include an adequately assessed clinical SAD sample. A number of experimental studies have utilised self-report measures, such as the Social Interaction Anxiety Scale (SIAS; Mattick \& Clark, 1998; e.g., Brozovich \& Heimberg, 2013; Judah et al., 2013; Kashdan \& Roberts, 2004), the Social Phobia Inventory (SPIN; Connor et al., 2000; e.g., Chiupka et al., 2012), or the Brief Fear of Negative Evaluation Scale (BFNE; Leary, 1983; e.g., Brown \& Stopa, 2006; Grisham et al., 2015; Makkar \& Grisham, 2013) to create analogue high and low social anxiety groups. While researchers believe that social anxiety exists on a continuum and social fears may cause functional impairment in individuals who do not meet full criteria for SAD (Stopa \& Clark, 2001), caution must be taken in the application of these findings to clinical populations. While a number of experimental studies have utilised appropriately assessed SAD samples in the study of SFA (e.g., Chen et al., 2013; Woody \& Rodriguez, 2000) and PER (e.g., Abbott \& Rapee, 2004; Zou \& Abbott, 2012), only one study to date has investigated AR in a clinical sample (Penney \& Abbott, 2015).

In addition, there is considerable variation in the methodology utilised to elicit state social anxiety across studies. Studies with strong ecological validity have employed a current social task, such as a speech (e.g., Makkar \& Grisham, 2013; Perini et al., 2012) or interaction (e.g., Furukawa et al., 2009; Zou \& Abbott, 2012), allowing for assessment of state affect and cognitive processes. However, other studies have assessed participants in a non-anxious state (e.g., Fisak \& Hammond, 2013; Vassilopoulos et al., 2015) or have asked participants to recall past social failures (e.g., Cassin \& Rector, 2011; Rachman et al., 2000; Wong et al., 2015), limiting the application of findings to situations involving current social threat. There is a particular paucity of strong experimental investigation of AR, with only one study of AR to date utilising a state social task (Penney \& Abbott, 2015).

Furthermore, while cognitive models of SAD postulate that self-focused cognitive processes are interdependent, there is almost no empirical evidence demonstrating that relationships exist among SFA, AR, and PER. Moreover, if these processes are connected, the nature of such relationships is poorly understood. All studies to date have focused on the impact of SFA on PER, and there is no empirical evidence demonstrating the possible impact of PER on AR, and AR on SFA. In addition, a small number of studies have begun to explore the neural underpinnings of SFA (Boehme et al., 2015; Judah et al., 2013); however, these findings are preliminary, and no studies to date have explored the neural correlates of AR and PER. Finally, the role of external focus of attention in the maintenance of SAD and its interaction with internal cognitive processes remains unclear. This is likely to be important to understanding the maintenance of SAD, and increasing the efficacy of interventions for the disorder.

\section{Summary and Future Research}

The current review has provided empirical support for the role of self-focused cognitive processes (SFA, AR, PER) in maintaining SAD, as proposed by cognitive models of the disorder. There is a substantial body of evidence demonstrating that socially 
anxious individuals engage in self-focused cognition during (SFA) and following (PER) a social or performance situation, and a smaller but growing body of literature suggesting that a similar process occurs prior (AR) to such situations. Furthermore, the vast majority of research to date indicates that self-focused cognitive processes are maladaptive, and maintain SAD in a variety of ways. However, there remains considerable scope for future research to further investigate the role of SFA, AR, and PER in the maintenance of SAD, and the development of efficacious interventions designed to ameliorate their negative effects.

Future research is required to address the methodological limitations in the field, including use of adequately assessed clinical SAD samples and ecologically valid social tasks, especially regarding AR. Research is needed to experimentally explore the relationships between SFA, AR and PER, as well as mediators of the relationship between $\mathrm{SAD}$ and these self-focused cognitive processes. Furthermore, the interaction between internal and external attentional focus requires direct investigation to test the predictions of the two key models of SAD (Clark \& Wells, 1995; Rapee \& Heimberg, 1997) with respect to the role of external attentional focus. Moreover, further studies are needed that utilise measures beyond self-report, including physiological arousal (e.g., Grisham et al., 2015) and eye-tracking (e.g., Judah et al., 2013), as well as functional magnetic resonance imaging (fMRI) to further investigate the neurological underpinning of self-focused cognitive processes in SAD. Additional research investigating these processes among socially anxious children and adolescents would also be valuable, allowing for greater understanding of the development of self-focused cognitive processes across the lifespan. While there is preliminary evidence supporting the role of SFA (Higa \& Daleiden, 2008; Hodson et al., 2008; Kley et al., 2011; Ranta et al., 2014) and PER (Schmitz et al., 2010; Schmitz et al., 2011) in socially anxious young people, no studies to date have investigated AR, and most research has employed analogue samples. Furthermore, it remains unclear whether the self-focused cognitive processes found among SAD individuals are transdiagnostic (e.g., Schmitz et al., 2011; McEvoy, Mahoney, \& Moulds, 2010; Rood, Roelofs, Bögels, \& Alloy, 2010) or specific to SAD and social threat (e.g., Abbott \& Rapee, 2004; Fehm, Schneider, \& Hoyer, 2007; Kocovski \& Rector, 2007; Wong et al., 2015), and this issue this is worthy of further investigation. Finally, further exploration of the role of imagery (see $\mathrm{Ng}$, Abbott, \& Hunt, 2014) and positive metacognitive beliefs in self-focused cognitive processes (Fisak \& Hammond, 2013; Vassilopoulos et al., 2015) is warranted, and may provide avenues for improving current treatments for SAD.

\section{Clinical Implications}

The findings of the current review have significant implications for the treatment of $\mathrm{SAD}$, suggesting that self-focused cognitive processes should be key targets for the amelioration of symptoms. Indeed, findings among SAD individuals that reductions in SFA mediate symptom reduction in CBT (e.g., Mörtberg et al., 2015) and that PER attenuates response to CBT (Price \& Anderson, 2011) indicate that these processes should be a central focus of treatment. However, interventions for reducing selffocused cognition among SAD individuals are often embedded within larger trials of CBT. While a small number of studies have attempted to isolate the effect of reducing SFA (e.g., Mörtberg et al., 2015; Schreiber et al., 2015) and PER (e.g., Price $\&$ Anderson, 2011), the hybrid nature of CBT generates difficulty in assessing the impact of specific therapeutic components. 
Nonetheless, a number of studies have demonstrated benefits of interventions aimed at reducing SFA, including Attention Training (AT; Wells, 1990) and Task Concentration Training (TCT; Bogels, Mulkens, \& De Jong, 1997). These interventions aim to increase flexibility within maladaptive attentional processes by reducing internal self-focus, increasing external or task-focused attention, increasing attentional breadth, and improving ability to shift attention between stimuli (Wells \& Papageorgiou, 1998; Wells, White, \& Carter, 1997). Several studies have found such training to be efficacious when practised in the context of a hierarchy of socially threatening situations, thus allowing exposure to social situations, as well as to somatic anxiety symptoms experienced in such situations (e.g., Bögels, 2006; Mulkens, Bögels, de Jong, \& Louwers, 2001; Härtling, Klotsche, Heinrich, \& Hoyer, 2015). However, Donald, Abbott, and Smith (2014) found AT to be effective independent of direct exposure to social situations, yielding significant reductions in SAD symptomatology as a result of training participants' ability to focus, strengthen, and shift attention in the context of interoceptive exercises.

Indeed, interoceptive exposure, a behavioural intervention aiming to reduce distress associated with somatic symptoms, has demonstrated transdiagnostic utility in the treatment of a range of disorders, including SAD (Boettcher, Brake, \& Barlow, 2015; Collimore \& Asmundson, 2014; Dixon, Kemp, Farrell, Blakey, \& Deacon, 2015). The use of interoceptive exposure to reduce anxiety sensitivity (distress associated with anxiety symptoms and their perceived consequences) among SAD individuals may diminish the deleterious impact of SFA, thereby interrupting the vicious cycle of anxiety. Specifically, Dixon and colleagues (2015) investigated a range of established and novel interoceptive exercises designed to elicit anxiety symptoms that are especially feared by SAD individuals (blushing, sweating, trembling). The authors report that: (1) blushing was most elicited by placing a heat pack on one's face and consuming hot sauce; (2) sweating was most elicited by placing a heat pack on one's face, running, and doing push-ups; and (3) trembling was most induced by push-ups and weight tasks. Interoceptive exposure exercises designed to optimise corrective learning and reduce idiosyncratically feared anxiety symptoms among SAD individuals may be valuable in disrupting the anxiety escalation typically initiated by SFA. Thus, attention training and interoceptive exposure interventions exhibit considerable promise for enhancing treatment outcomes, and evidence supports their integration into standard treatment protocols for SAD (Boettcher et al., 2015; McEvoy \& Perini, 2009).

In addition, cognitive strategies and behavioural experiments aiming to discourage the use of AR and PER have demonstrated utility in the treatment of SAD (e.g., Clark, 2001; Clark et al., 2003; Clark et al., 2006). However, mindfulness and acceptancebased strategies may also be of benefit in reducing the impact self-focused rumination among SAD individuals, as has been demonstrated for depressive rumination (e.g., van Aalderen, Donders, Peffer, \& Spckens, 2015). It is argued that mindfulness and acceptance-based strategies facilitate a decentred perspective on thoughts and feelings, which are observed as subjective and transient events, rather than necessarily accurate reflections of truth or reality that compel specific behaviours (Bishop et al., 2004). This decentred perspective is hypothesised to reduce rumination and automatic reacting to experience, thereby enabling more reflective, flexible responding to situations. Indeed, mindfulness and acceptance-based interventions have yielded significant reductions in symptomatology across treatment studies for SAD, suggesting that they may be a viable intervention for the disorder (see Norton, Abbott, Norberg, $\&$ Hunt, 2015, for a systematic review). Specifically, studies of SAD individuals have 
found a brief mindfulness intervention following a public speaking task reduces PER and associated negative affect significantly more than a control condition (Shikatani, Antony, Kuo, \& Cassin, 2014), and engaging mindfully with experience during a PER induction reduces distress and increases net positive affect significantly more than a control procedure (Cassin \& Rector, 2011). Hence, the integration of mindfulness and acceptance-based interventions for reducing the impact of rumination before and after social situations among may be a useful avenue of investigation for augmenting the treatment of SAD.

\section{Acknowledgments}

This research received no specific grant from any funding agency, commercial or not-for-profit sectors.

\section{Declaration of Interest}

None.

\section{References}

Abbott, M., \& Rapee, R. (2004). Post-event rumination and negative self-appraisal in social phobia before and after treatment. Journal of Abnormal Psychology, 113, 136-144.

American Psychiatric Association. (2013). Diagnostic and statistical manual of mental disorders (5th ed.). Washington, DC: Author.

Australian Bureau of Statistics (ABS). (2007). National survey of mental health and wellbeing: Summary of results. Canberra, Australian Capital Territory: Author. Retrieved from http://www.abs.gov.au/AUSSTATS/abs@.nsf/mf/4326.0

Beidel, D., Turner, S., \& Dancu, C. (1985). Physiological, cognitive and behavioral aspects of social anxiety. Behaviour Research and Therapy, 23, 109-117.

Bishop, S., Lau, M., Shapiro, S., Carlson, L., Anderson, N., Carmody, J., ... Devins, G. (2004). Mindfulness: A proposed operational definition. Clinical Psychology: Science and Practice, 11, 230-241.

Blagden, J., \& Craske, M. (1996). Effects of active and passive rumination and distraction: A pilot replication with anxious mood. Journal of Anxiety Disorders, 10, 243-252.

Boehme, S., Miltner, W., \& Staube, T. (2015). Neural correlates of self-focused attention in social anxiety. Scan, 10, 856-862.

Boettcher, H., Brake, A., \& Barlow, D. (2015). Origins and outlook of interoceptive exposure. Journal of Behavior Therapy and Experimental Psychiatry. Advance online publication.

Bögels, S. (2006). Task concentration training versus applied relaxation, in combination with cognitive therapy, for social phobia patients with fear of blushing, trembling, and sweating. Behaviour Research and Therapy, 44, 119-1210.

Bögels, S., \& Mansell, W., (2004). Attention processes in the maintenance and treatment of social phobia: Hyperbigilance, avoidance, and self-focused attention. Clinical Psychology Review, 24, $827-856$.

Bögels, S., Mulkens, S., \& de Jong, P. (1997). Task concentration training and fear of blushing. Journal of Clinical Psychology and Psychotherapy, 4, 251-258.

Bögels, S., Rijsemus, W., \& De Jong, P. (2002). Self-focused attention and social anxiety: The effects of experimentally heightened self-awareness on fear, blushing, cognitions, and social skills. Cognitive Therapy and Research, 26, 461-472.

Brown, M., \& Stopa, L. (2006). Does anticipation help or hinder performance in a subsequent speech? Behavioural and Cognitive Psychotherapy, 35, 133-147. 
Brozovich, F., \& Heimberg, R. (2008). An analysis of post-event processing in social anxiety disorder. Clinical Psychology Review, 28, 891-903.

Brozovich, F., \& Heimberg, R. (2013). Mental imagery and post-event processing in anticipation of a speech performance among socially anxious individuals. Behavior Therapy, 44, 701-716.

Burgio, K., Merluzzi, T., \& Pryor, J. (1986). Effects of performance expectancy and self-focused attention on social interaction. Journal of Personality and Social Psychology, 50, 1216-1221.

Cassin, E., \& Rector, N. (2011). Mindfulness and the attenuation of post-event processing in social phobia: An experimental investigation. Cognitive Behvaviour Therapy, 40(4), 267-278.

Chen, J., Rapee, R., \& Abbott, M. (2013). Mediators of the relationship between social anxiety and post-event rumination. Journal of Anxiety Disorders, 27, 1-8.

Chiupka, C., Mosovitch, D., \& Bielak, T. (2012). In vivo activa tion of anticipatory vs. post-event autobiographical images and memories in social anxiety. Journal of Social and Clinical Psychology, 31, 783-809.

Clark, D. (2001). A cognitive perspective on social phobia. In W. R. Crozier, \& L. E. Alden (Eds.), International handbook of social anxiety: Concepts, research and interventions relating to the self and shyness (pp. 405-430). New York: John Wiley \& Sons.

Clark, D.M., Ehlers, A., Hackmann, A., McManus, F., Fennell, M., Grey, N., ... Wild, J. (2006). Cognitive therapy versus exposure \& applied relaxation in SAD: A randomized controlled trial. Journal of Consulting and Clinical Psychology, 74, 568-578.

Clark, D.M., Ehlers, A., McManus, F., Hackmann, A., Fennell, M., Campell, H., ... Louis, B. (2003). Cognitive therapy vs. fluoxetine plus self exposure in the treatment of generalized SAD (social anxiety disorder): A randomized placebo controlled trial. Journal of Consulting and Clinical Psychology, 71, 1058-1067.

Clark, D., \& Wells, A. (1995). A cognitive model of social phobia. In R. Heimberg, M. Liebowitz, D. Hope, \& R. Schneier (Eds.), Social phobia: Diagnosis, assessment and treatment (pp. 69-93). New York: Guilford Press.

Coles, M., Turk, C., \& Heimberg, R. (2002). The role of memory perspective in social phobia: Immediate and delayed memories for role-played situations. Behavioural and Cognitive Psychotherapy, $30,415-425$.

Coles, M., Turk, C., Heimberg, R., \& Fresco, D. (2001). Effects of varying levels of anxiety within social situations: Relationships to memory perspective and attributions in social phobia. Behaviour Research and Therapy, 39, 651-665.

Collimore, K., \& Asmundson, G. (2014). Fearful responding to interoceptive exposure in social anxiety disorder. Journal of Anxiety Disorders, 28, 195-202.

Connor, K., Davidson, J., Churchill, L., Sherwood, A., Foa, E., \& Weisler, R. (2000). Psychometric properties of the social phobia inventory (SPIN): New self-rating scale. British Journal of Psychiatry, 176, 379-386.

Daly, J., Vangelisti, A., \& Lawrence, S. (1989). Self-focused attention and public speaking anxiety. Personality and Individual Differences, 10, 903-913.

Dannahy, L., \& Stopa, L. (2007). Post-event processing in social anxiety. Behaviour Research and Therapy, 45, 1207-1219.

Dixon, L., Kemp, J., Farrell, N., Blakey, S., \& Deacon, B. (2015). Interoceptive exposure exercises for social anxiety. Journal of Anxiety Disorders, 33, 25-34.

Dodge, C., Hope, D., Heimberg, R., \& Becker, R. (1988). Evaluation of the Social Interaction Self-Statement Test in a social phobic population. Cognitive Therapy and Research, 12, 211-222.

Donald, J., Abbott, M., \& Smith, E. (2014). Comparison of attention training and cognitive therapy in the treatment of social phobia. Behavioural and Cognitive Psychotherapy, 42, 74-91.

Edwards, S., Rapee, R., \& Franklin, J. (2003). Post-event rumination and recall bias for social performance event in high and low socially anxious individuals. Cognitive Therapy and Research, $27,603-617$.

Fehm, L., \& Margraf, J. (2002). Thought suppression: Specificity in agoraphobia versus broad impairment in social phobia? Behaviour Research and Therapy, 40, 57-66. 
Fehm, L., Schneider, G., \& Hoyer, J. (2007). Is post-event processing specific for social anxiety? Journal of Behavior Therapy and Experimental Psychiatry, 38, 11-22.

Field, A., \& Morgan, J. (2004). Post-event processing and the retrieval of autobiographical memories in socially anxious individuals. Journal of Anxiety Disorders, 18, 647-663.

Fisak, B., \& Hammond, A. (2013). Are positive beliefs about post-event processing related to social anxiety? Behaviour Change, 30, 36-47.

Furukawa, T., Chen, J., Watanabe, N., Nakano, Y., Ietsugu, T., Ogawa, S., Funayama, T., \& Noda, Y. (2009). Videotaped experiments to drop safety behaviors and self-focused attention for patients with social anxiety disorder: Do they change subjective and objective evaluations of anxiety and performance? Journal of Behavior Therapy and Experimental Psychiatry, 40, 202-210.

Gaydukevych, E., \& Kocovski, N. (2012). Effect of self-focused attention on post-event processing in social anxiety. Behaviour Research and Therapy, 50, 47-55.

Gramer, M., Schild, E., \& Lurz, E. (2012). Objective and perceived physiological arousal in trait social anxiety and post-event processing of a prepared speaking task. Personality and Individual Differences, 53, 980-984.

Grisham, J., King, B., Makkar, S., \& Felmingham, K. (2015). The contributions of arousal and self-focused attention to avoidance in social anxiety. Anxiety, Stress, $\mathcal{E}$ Coping, 28, 303-320.

Hackmann, A., Clark, D., \& McManus, F. (2000). Recurrent images and early memories in social phobia. Behaviour Research and Therapy, 38, 601-610.

Hackmann, A., Surawy, C., \& Clark, D. (1998). Seeing yourself through others' eyes: A study of spontaneously occurring images in social phobia. Behavioural and Cognitive Psychotherapy, 26, $3-12$.

Härtling, S., Klotsche, J., Heinrich, A., \& Hoyer, J. (2015). Cognitive therapy and task concentration training applied as intensified group therapies for social anxiety disorder with fear of blushing A randomized controlled trial. Clinical Psychology and Psychotherapy.

Hayes, S., Wilson, K., Strosahl, K., Gifford, E., \& Follette, V. (1996). Experiential avoidance and behavioral disorders: A functional dimensional approach to diagnosis and treatment. Journal of Consulting and Clinical Psychology, 64, 1152-1168.

Hedman, E., Mörtberg, E., Hesser, H., Clark, D., Lekander, M., Anderson, E., \& Ljótsson, B. (2013). Mediators in psychological treatment of social anxiety disorder: Individual cognitive therapy compared to cognitive behavioral group therapy. Behaviour Research and Therapy, 51, 696-705.

Heimberg, R., Bozovich, F., \& Rapee, R. (2010). A cogntive behavioral model of social anxiety disorder: Update and extension. In S.G. Hofmann \& P.M. DiBartolo (Eds.), Social anxiety: Clinical, developmental, and social perspective (pp. 395-422). Saint Louis: Elsevier Science.

Heimberg, R., Bruch, M., Hope, D., \& Dombeck, M. (1990). Evaluating the States of Mind model: Comparison to an alternative model and effects of method of cognitive assessment. Cognitive Therapy and Research, 14, 543-557.

Higa, C., \& Daleiden, E. (2008). Social anxiety and cognitive biases in non-referred children: The interaction of self-focused attention and threat interpretation biases. Journal of Anxiety Disorders, $22,441-452$.

Hinrichsen, H., \& Clark, D. (2003). Anticipatory processing in social anxiety: Two pilot studies. Journal of Behavior Therapy and Experimental Psychiatry, 34, 205-218.

Hirsch, C., Clark, D., Mathews, A., \& Williams, R. (2003). Self-images play a causal role in social phobia. Behaviour Research and Therapy, 41, 909-921.

Hodson, K., McManus, F., Clark, D., \& Doll, H. (2008). Can Clark and Wells' (1995) cognitive model of social phobia be applied to young people? Behavioural and Cognitive Psychotherapy, 26, $449-461$.

Hofmann, S. (2007). Cognitive factors that maintain social anxiety disorder: A comprehensive model and its treatment implications. Cognitive Behaviour Therapy, 36, 193-209.

Holzman, J., Valentiner, D., \& McCraw, K. (2014). Self-focused attention and post-event processing: Relevance to social performance anxiety and social interaction anxiety. Journal of Cognitive Psychotherapy, 28, 72-82. 
Hope, D., \& Heimberg, R. (1988). Public and private self-consciousness and social phobia. Journal of Personality Assessment, 52, 626-639.

Hope, D., Gansler, D., \& Heimberg, R. (1989). Attentional focus and causal attributions in social phobia: Implications from social psychology. Clinical Psychology Review, 9, 49-60.

Hope, D., Heimberg, R., \& Klein, J. (1990). Social anxiety and the recall of interpersonal information. Journal of Cognitive Psychotherapy: An International Quarterly, 4, 185-195.

Ingram, R. (1990). Self-focused attention in clinical disorders: Review and conceptual model. Psychological Bulletin, 107, 156-176.

Judah, M., Grant, D., Mills, A., \& Lechner, W. (2013). The neural correlates of impaired attentional control in social anxiety: an ERP study of inhibition and shifting. Emotion, 13, 1096-1106.

Kashdan, T., \& Roberts, J. (2004). Social anxiety's impact on affect, curiosity, and social self-efficacy during a high self-focus social threat situation. Cognitive Therapy and Research, 28, 119-141.

Kiko, S., Stevens, S., Mall, A., Steil, R., Bohus, M., \& Hermann, C. (2012). Predicting post-event processing in social anxiety disorder following two prototypical social situations: State variables and dispositional determinants. Behaviour Research and Therapy, 50, 617-626.

Kley, H., Tuschen-Caffier, B., \& Heinrichs, N. (2011). Manipulating self-focused attention in children with social anxiety disorder and in socially anxious and non-anxious children. Journal of Experimental Psychopathology, 2, 551-570.

Kocovski, N., Endler, N., Rector, N., \& Flett, G. (2005). Rumination and postevent processing in social anxiety. Behaviour Research and Therapy, 43, 971-984.

Kocovski, N., MacKenzie, M., \& Rector, N. (2011). Rumination and distraction periods immediately following a speech task: Effect on postevent processing in social anxiety. Cognitive Behaviour Therapy, 40, 45-56.

Kocovski, N., \& Rector, N. (2007). Predictors of post-event rumination related to social anxiety. Cognitive Behaviour Therapy, 43, 1-11.

Kocovski, N., \& Rector, N. (2008). Post-event processing in social anxiety disorder: Idiosyncratic priming in the course of CBT. Cognitive Therapy and Research, 32, 23-36.

Leary, M. (1983). A brief version of the Fear of Negative Evaluation Scale. Personality and Social Psychology Bulletin, 9, 371-375.

Lehrer, P., \& Woolfolk, R. (1982). Self-report assessment of anxiety: Somatic, cognitive, and behavioral modalities. Behavioral Assessment, 4, 167-177.

Lundh, L., \& Sperling, M. (2002). Social anxiety and the post-event processing of socially distressing events. Cognitive Behaviour Therapy, 31, 129-134.

Mahone, E., Bruch, M., \& Heimberg, R. (1993). Focus of attention and social anxiety: The role of negative self-thoughts and perceived positive attributes of the other. Cognitive Therapy and Research, 17, 209-224.

Makkar, S., \& Grisham, J. (2011a). The predictors and contents of post-event processing in social anxiety. Cognitive Therapy and Research, 35, 118-133.

Makkar, S., \& Grisham, J. (2011b). Social anxiety and the effects of negative self-imagery on emotion, cognition, and post-event processing. Behaviour Research and Therapy, 49, 654-664.

Makkar, S., \& Grisham, J, (2013). Effects of false feedback on affect, cognition, behavior, and postevent processing: the mediating role of self-focused attention. Behavior Therapy, 44, 111124.

Mansell, W., Clark, D., \& Ehlers, A. (2003). Internal versus external attention in social anxiety: An investigation using a novel paradigm. Behaviour Research and Therapy, 41, 555-572.

Mattick, R., \& Clarke, J. (1998). Development and validation of measures of social phobia scrutiny fear and social interaction anxiety. Behaviour Research and Therapy, 36, 455-470.

McEvoy, P., Mahoney, A., \& Moulds, M. (2010). Are worry, rumination, and post-event processing one and the same? Development of the repetitive thinking questionnaire. Journal of Anxiety Disorders, 24, 509-519.

McEvoy, P., \& Perini, S. (2009). Cognitive behavioral group therapy for social phobia with or without attention training: A controlled trial. Journal of Anxiety Disorders, 23, 519-528. 
Mellings, T., \& Alden, L. (2000). Cognitive processes in social anxiety: the effects of self-focus, rumination and anticipatory processing. Behaviour Research and Therapy, 38, 243-257.

Mills, A., Grant, D., Judah, M., \& White, E, (2014). The influence of anticipatory processing on attentional biases in social anxiety. Behavior Therapy, 45, 720-729.

Mills, A., Grant, D., Lechner, W., \& Judah, M. (2014). Relationship between trait repetitive negative thinking styles and symptoms of psychopathology. Personality and Individual Differences, 71, 1924.

Mörtberg, E., Hoffart, A., Boecking, B., \& Clark, D. (2015). Shifting the focus of one's attention mediates improvement in cognitive therapy for social anxiety disorder. Behavioural and Cognitive Psychotherapy, 43, 63-73.

Mulkens, S., Bögels, S., de Jong, P., \& Louwers, J. (2001). Fear of blushing: Effectos of task concentration training in vivo on fear and physiology. Anxiety Disorders, 15, 413-432.

Ng, A., Abbott, M., \& Hunt, C. (2014). The effect of self-imagery on symptoms and processes in social anxiety: A systematic review. Clinical Psychology Review, 34, 620-633.

Nolen-Hoeksema, S., Wisco, B., \& Lyubomirsky, S. (2008). Rethinking rumination. Perspectives on Psychological Science, 3, 400-424.

Norton, A., Abbott, M., Norberg, M., \& Hunt, C. (2015). A systematic review of mindfulness and acceptance-based treatments for social anxiety disorder. Journal of Clinical Psychology, 71, 283-301.

Norton, P., \& Hope, D. (2001). Kernels of truth or distorted perceptions: Self and observer ratings of social anxiety and performance. Behavior Therapy, 32, 765-786.

Penney, E., \& Abbott, M., (2015). The impact of perceived standards on state anxiety, appraisal processes, and negative pre- and post-event rumination in social anxiety disorder. Cognitive Therapy and Research, 39, 162-177.

Perini, S., Abbott, M., \& Rapee, R. (2006). Perception of performance as a mediator in the relationship between social anxiety and negative post-event rumination. Cognitive Therapy and Research, $30,645-659$.

Pineles, S., \& Mineka, S. (2005). Attentional bias to internal and external sources of potential threat in social anxiety. Journal of Abnormal Psychology, 114, 314-318.

Pozo, C., Carver, C., Wellens, A., \& Scheier, M. (1991). Social anxiety and social perception: Construing others' reactions to the self. Personality and Social Psychology Bulletin, 17, 355-362.

Price, M., \& Anderson, P. (2011). The impact of cognitive behavioral therapy on post event processing among those with social anxiety disorder. Behaviour Therapy and Research, 49, 132 137.

Rachman, S., Grüter-Andrew, J., \& Shafran, R. (2000). Post-event processing in social anxiety. Behaviour Research and Therapy, 38, 611-617.

Ranta, K., Tuomisto, M., Kaltiala-Heino, R., Rantanen, P., \& Marrunen, M. (2014). Cognition, imagery and coping among adolescents with social anxiety and phobia: Testing the clark and wells model in the population. Clinical Psychology and Psychotherapy, 21, 252-263.

Rapee, R., \& Heimberg, R. (1997). A cognitive-behavioral model of anxiety in social phobia. Behaviour Research and Therapy, 35, 741-756.

Rood, L., Roelofs, J., Bögels, S., \& Alloy, L. (2010). Dimensions of negative thinking and the relations with symptoms of depression and anxiety in children and adolescents. Cognitive Therapy and Research, 34, 333-342.

Salters-Pedneault, K., Tull, M., \& Roemer, L. (2004). The role of avoidance of emotional material in the anxiety disorders. Applied and Preventive Psychology, 11, 95-114.

Schmitz, J., Krämer, M., Blechert, J., \& Tuschen-Caffier, B. (2010). Post-event processing in children with social phobia. Journal of Abnormal Child Psychology, 38, 911-919.

Schmitz, J., Krämer, M., \& Tuschen-Caffier, B. (2011). Negative post-event processing and decreased self-appraisals of performance following social stress in childhood social anxiety: An experimental study. Behaviour Research and Therapy, 49, 789-795. 
Schreiber, F., Heimlich, C., Schweitzer, C., \& Stangier, U. (2015). Cognitive therapy for social anxiety disorder: The impact of the 'self-focused attention and safety behaviours experiment' on the course of treatment. Behavioural and Cognitive Psychotherapy, 43, 158-166.

Schultz, L., \& Heimberg, R. (2008). Attentional focus in social anxiety disorder: Potential for interactive processes. Clinical Psychology Review, 28, 1206-1221.

Shikatani, B., Antony, M., Kuo, J., \& Cassin, S. (2014). The impact of cognitive restructuring and mindfulness strategies on postevent processing and affect in social anxiety disorder. Journal of Anxiety Disorders, 28, 570-579.

Spurr, J., \& Stopa, L. (2002). Self-focused attention in social phobia and social anxiety. Clinical Psychology Review, 22, 947-975.

Spurr, J., \& Stopa, L. (2003). The observer perspective: Effects on social anxiety and performance. Behaviour Research and Therapy, 41, 1009-1028.

Stein, M., \& Stein, D. (2008). Social anxiety disorder. Lancet, 371, 1115-1125.

Stopa, L., \& Clark, D. (1993). Cognitive processes in social phobia. Behaviour Research and Therapy, 31, 255-267.

Stopa, L., \& Clark, D. (2001). Social phobia: Comments on the viability and validity of an analogue research strategy and British norms for the fear of negative evaluation questionnaire. Behavioural and Cogntive Psychotherapy, 29, 423-430.

Thomsen, D. (2006). The association between rumination and negative affect: A review. Cognition and Emotion, 20, 1216-1235.

van Aalderen, J., Donders, A., Peffer, K., \& Spekens, A. (2015). Long-term outcome of mindfulnessbased cognitive therapy in recurrently depressed patients with and without a depressive episode at baseline. Depression and Anxiety, 32, 563-569.

Vassilopoulos, S. (2004). Anticipatory processing in social anxiety. Behavioural and Cognitive Psychotherapy, 32, 285-293.

Vassilopoulos, S. (2005). Anticipatory processing plays a role in maintaining social anxiety. Anxiety, Stress, and Coping, 18, 321-332.

Vassilopoulos, S. (2008). Coping strategies and anticipatory processing in high and low socially anxious individuals. Journal of Anxiety Disorders, 22, 98-107.

Vassilopoulos, S., Brouzos, A., \& Moberly, N. (2015). The relationships between metacognition, anticipatory processing, and social anxiety. Behaviour Change, 32, 114-126.

Wang, P., Lane, M., Olfson, M., Pincus, H., Wells, K., \& Kessler, R. (2005). Twelve-month use of mental health services in the United States: Results from the National Comorbidity Survey Replication. Archives of General Psychiatry, 62, 629-40.

Watkins, E., \& Teasdale, J. (2004). Adaptive and maladaptive self-focus in depression. Journal of Affective Disorders, 82, 1-8.

Wells, A. (1990). Panic disorder in association with relaxation induced anxiety: An attentional training approach to treatment. Behavior Therapy, 21, 273-280.

Wells, A., \& Clark, D.M. (1997). Social phobia: A cognitive approach. In G.C.L. Davey (Ed.), Phobias: A handbook of theory, research and treatment (pp. 3-26). Chichester, UK: Wiley.

Wells, A., \& Papageorgiou, C. (1998). Social phobia: Effects of external attention on anxiety, negative beliefs, and perspective taking. Behavior Therapy, 29, 357-370.

Wells, A., \& Papageorgiou, C. (2001). Social phobic interoception: Effects of bodily information on anxiety, beliefs and self-processing. Behaviour Research and Therapy, 39, 1-11.

Wells, A., White, J., \& Carter, K. (1997). Attention training: Effects on anxiety and beliefs in panic and social phobia. Clinical Psychology and Psychotherapy, 4, 226-232.

Wong, Q., \& Moulds, M. (2009). Impact of rumination versus distraction on anxiety and maladaptive self-beliefs in socially anxious individuals. Behaviour Research and Therapy, 47, 861-867.

Wong, Q., \& Moulds, M. (2011). Impact of anticipatory processing versus distraction on multiple indices of anxiety in socially anxious individuals. Behaviour Research and Therapy, 49, 700706. 
Wong, Q., McEvoy, P., \& Rapee, R. (2015). A comparison of repetitive negative thinking and post-event processing in the prediction of maladaptive social-evaluative beliefs: A short-term prospective study. Journal of Psychopathology and Behavioural Assessment, published online.

Woody, S. (1996). Effects of focus of attention on anxiety levels and social performance of individuals with social phobia. Journal of Abnormal Psychology, 105, 61-69.

Woody, S., \& Rodriguez, B. (2000). Self-focused attention and social anxiety in social phobics and normal controls. Cognitive Therapy and Research, 24, 473-488.

Zou, J., \& Abbott, M. (2012). Self-perception and rumination in social phobia: The impact of positive versus ambiguous feedback. Behaviour Research $\mathcal{E}$ Therapy, 50, 250-257.

Zou, J., Hudson, J., \& Rapee, R. (2007). The effect of attentional focus on social anxiety. Behavior Research and Therapy, 45, 2326-2333. 